\title{
Power reduction optimization with swarm based technique in electric power assist steering system
}

\begin{abstract}
Energy management in electric vehicle technology is very important as the energy source of all its system operations are solely relying on the battery. Efforts are being made to reduce the energy consumed as much as possible in electric vehicle system. As one of the auxiliary elements of the system, the electric power assist steering system can be controlled or manipulated in such a way that minimum energy from the battery source is being drawn during operation. This unique feature enables the system to be tuned with the optimal performance setting so that less power is needed for its optimum operation. The research's aim is to apply the swarm optimization technique; Particle Swarm Optimization and Ant Colony Optimization to improve the controller's performance. The investigation covers an analysis of power consumption for the system in simulation and experimental setup. Both simulation and experimental tests are conducted to validate the proposed controller performance in optimizing power reduction. It is proven that the ant colony optimization tuned controller outperform the controller tuning using particle swarm optimization for power minimization.
\end{abstract}

Keyword: Electric power assist steering system; Particle swarm optimization; Ant colony optimization; Electric vehicle 